\title{
Development of Microcontroller based Automated Public Transport Ticketing System
}

\author{
Telugu Maddileti, R.K. Pongiannan, V. Kranthi Sai Reddy, V. Sai Jahnavi, S. Prathima
}

\begin{abstract}
In India, generally printed tickets are issued for all kinds of transportation, and the printed tickets become useless once the passenger reaches their destination. For multiple modes of transportation like Bus, Trains, Metros, a multiple tickets are printed as well as a human verification is also required. Furthermore, it leads to financial loss and wastage of papers. In order to reduce the financial loss, time and human resource, a microcontroller based automated public transport ticketing system has been developed using a smart card and IoT. This ticketing system can be used to manage the billing in any mode of transportation like Bus, Trains, Metros, and Cabs. The automated public transport ticketing system is developed using a microcontroller and a single smart card which can be used for multiple transportation.
\end{abstract}

Keywords- Ticketing System, Smart Card, Mode of transportation

\section{INTRODUCTION}

In the past decades a mass transit systems have existed. In the traditional payment systems, tickets, cards and tokens are used for public transportation. In practice, the conventional paper printing based ticketing system is employed in most of the transportation in our country. In public road Transportation, the printed form of passenger tickets are issued. Also, the toll entry tickets are partially automated. The automation of transportation involves electronic processor, GPS, GSM, and IoT depending on the nature of the transport system. Mainly for the identification the wireless devices like RF, GPS and GSM are used. In our day to day activities, e-commerce applications are very popular in applications like e-ticket applications.

The electronic based transport ticketing system was developed for general transport [1] and railways [2]. Some Smart cards are secure, compact and intelligent data carriers. Smart cards should be regarded as specialized computers capable of processing, storing and safeguarding thousands of bytes of data. [3]. The wireless devices like RFID and smart card based system are reported in [4-8]. A complete infrastructure for public transportation using a novel server-based fare calculation and user management system was developed [9].

In this paper, an automated public transport ticketing system using Radio Frequency Identification (RFID) technology was developed.

Revised Version Manuscript Received on 10, September 2019. Telugu Maddileti, Department of ECM, SNIST, Telangana, India India.

V. Sai Jahnavi, Department of ECM, SNIST, Telangana, India.

S. Prathima, Department of ECM, SNIST, Telangana, India. R.K. Pongiannan, Dept. of EEE, SRMIST, Kancheepuram, Tamilnadu,

V. Kranthi Sai Reddy, Department of ECM, SNIST, Telangana, India.

\section{EXISTING SYSTEM}

In the existing transport ticketing system, an authenticated person collects the fares and issues the ticket in printed form and his responsibility is to make sure that every passenger has purchased the ticket. The main drawback is that, the passengers must carry the ticket until they reach their destination and also the passengers should always carry money. If a passenger wants to travel in multiple modes of public transportation, a multiple tickets are required. This kind of system in operation requires man power and incurs financial losses for papers. Moreover, the reliability and accuracy has to be improved.

\section{AUTOMATED PUBLIC TRANSPORT TICKETING SYSTEM}

Automated Public Transport Ticketing System is developed using a micro controller and a single smart card and the block schematic is shown in Fig.1. the automatic ticketing system consists of Arduino Nano, RFID Smart Card, Wi-Fi Module, Display unit, Switch. The application software has been developed using Arduino IDE

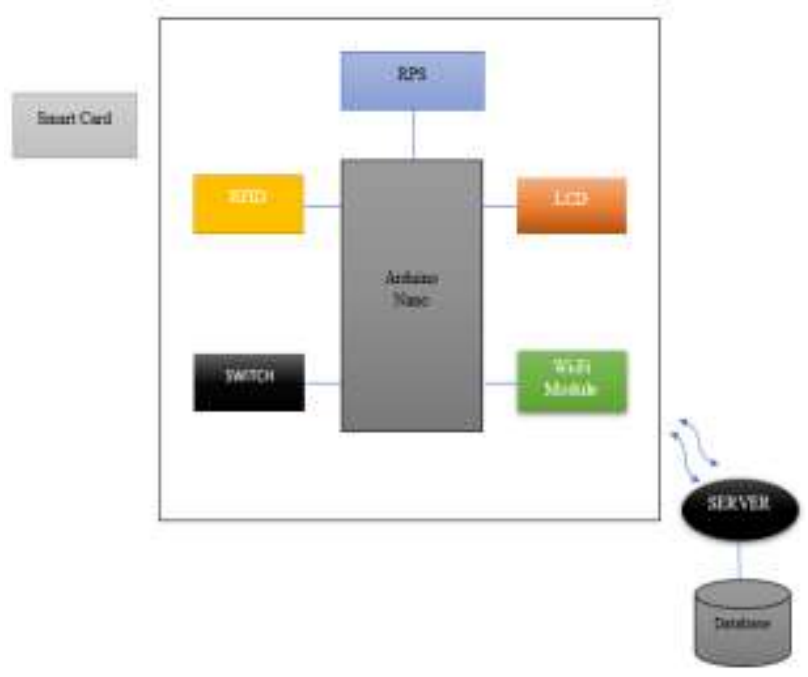

Fig 1: Block Diagram of microcontroller based automated public transport ticketing system

Arduino Nano Microcontroller- ATmega328p consists of 12 digital pins and 8 analog input-output pins. It can perform 3 types of communication protocols which are: Serial, SPI and $\mathrm{I} 2 \mathrm{C}$ protocol [10]. The programming is developed using Embedded C and Arduino IDE [11]. 


\section{DEVELOPMENT OF MICROCONTROLLER BASED AUTOMATED PUBLIC TRANSPORT TICKETING SYSTEM}

A set of four switches are used to select the four modes of transportation namely bus, cabs, trains and metros. Based on transportation, the respective switch is turned on. EM-18 RFID Reader[12] is used to read $125 \mathrm{kHz}$ RFID tags. This RFID Reader reads the smart card and send to the Arduino Nano. The microcontroller validates the smart card. A Liquid Crystal Display (LCD) screen display is used to display the information or messages.

ESP8266 Wi-Fi [13] Module is used to transfer the data or information regarding the passenger's day to day rides, amount credited and remaining balance in the smart card from device to user webpage. MySQL server manages the database regarding the Smart Card tag numbers and user profile. The webpage is coded in PHP language [14]. This website displays three actions namely view mode of transportation, recharge balance and logout action. When user clicks view mode of transportation, it displays all the rides travelled and the amount credited for each ride. The option is provided to check the balance in the smart card.

\section{A. System Description}

The experimental model of the automatic ticketing system developed is shown in Fig.2. The major elements of the system are the microcontroller, RFID reader, and a server. When a Smart Card is placed on RFID Reader, it reads and validate the smart card details are stored in the database server. After authentication, it requests the passenger to select the destination. The device checks the balance in the card, and allows passengers to Check-in. After passenger reaches the destination the Check-out process is initiated. When the passenger place the smart card on the RFID reader, the device check whether the passenger is checking out in same destination on every mode of public transportation vehicle. These devices are connected to back-end server. After the completion of every ride the data or information is sent to the webpage. The website is written in PHP code.

The website page has three operations one is view mode of transportation, recharge balance and another one is logout. When we click view mode of transportation, it will show all transportation details in which we travelled like buses, trains, cabs. The recharge balance is an option used to recharge the smart card and finally logout option is used to exit from the admin web-page. The travelling and transportation details are maintained by MySQL information.

The RFID consists of two elements one is RFID tag and the other is active type RFID Reader. RFID tag is the electronic tag and it exchanges the data within the RFID reader through Radio Waves. In the RFID tag 12 digits of data is stored. RFID reader is used to gather the information from RFID tag when it reaches the range i.e. billing station.

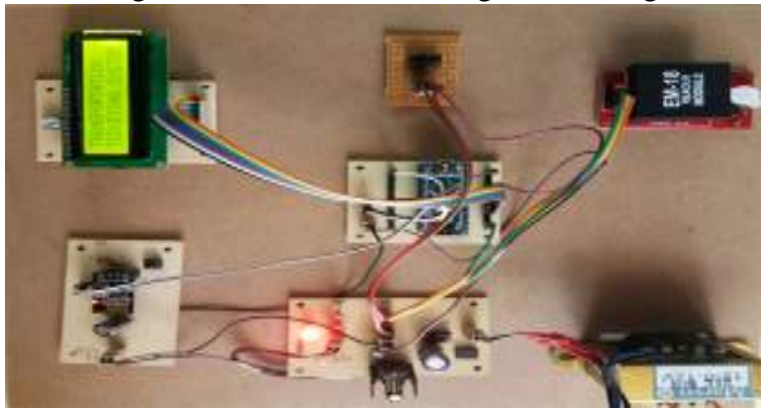

Fig 2: Experimental set up of the automatic ticketing system

\section{B. UML Diagrams}

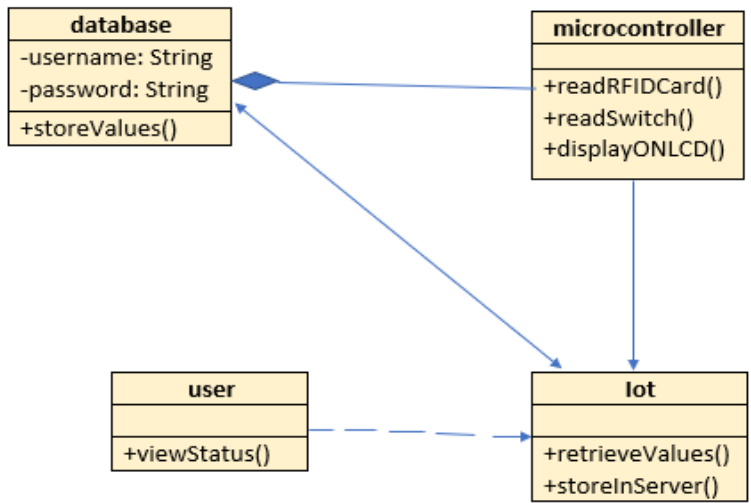

Fig 3: Class Diagram

Fig. 3 shows the class diagram. In this class diagram the symbol negative( $(-)$ is denoted as private and positive $(+)$ is known as public. Since the username and password are always private, the negative(-) symbol is used. The Fig.4 represents use case diagram having three major elements namely Microcontroller, IOT and User. Microcontroller performs three actions first it reads the RFID card and reads the switch i.e., mode of transportation and then displays on LCD screen. Now IOT performs two actions of retrieving the values and store in server.

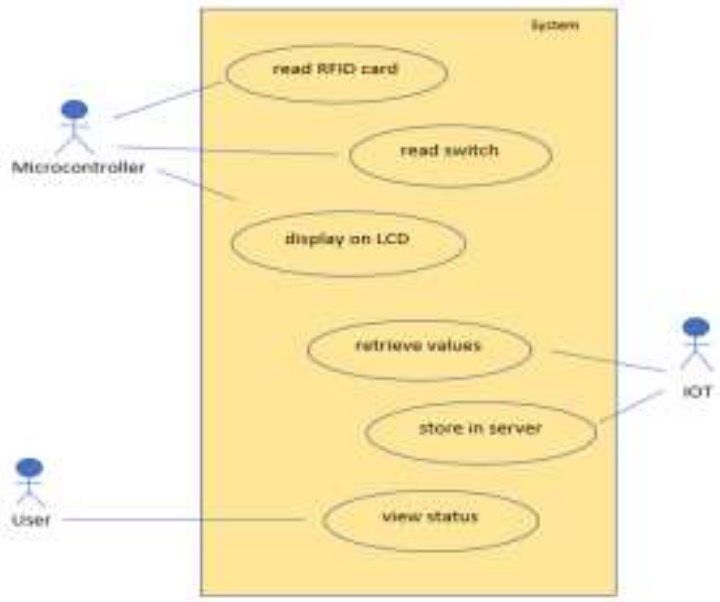

Fig 4: Use Case Diagram

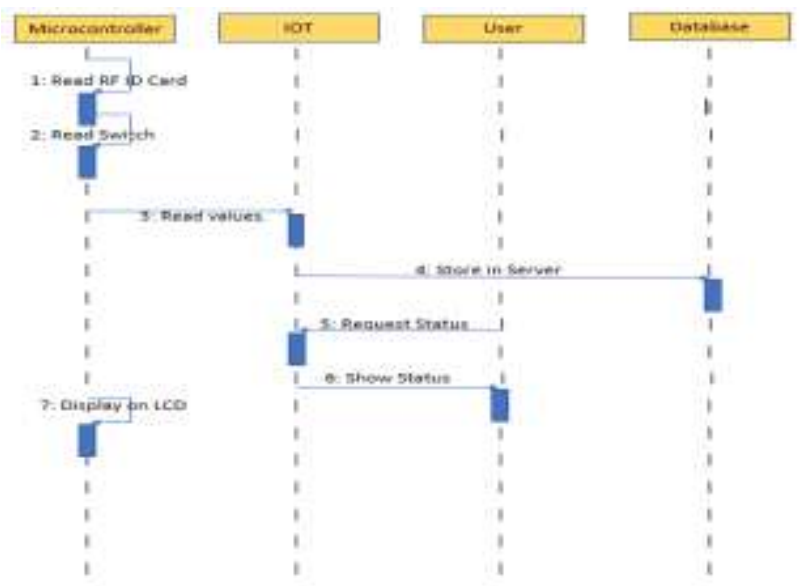

Fig 5: Sequence Diagram

Published By: Blue Eyes Intelligence Engineering \& Sciences Publication 
The Sequence Diagram shown in Fig.5 is used to depict the interaction between Microcontroller, IOT, User and Database in a sequential order. Microcontroller reads the smart card and switch, and it interacts with IOT to retrieve the values. The IOT interacts with Database to store in sever when user interacts with IOT to request the status then IOT interacts with user the show the status and finally microcontroller displays on LCD screen. The Fig. 7 shows the flow Chart of the automatic ticketing system.

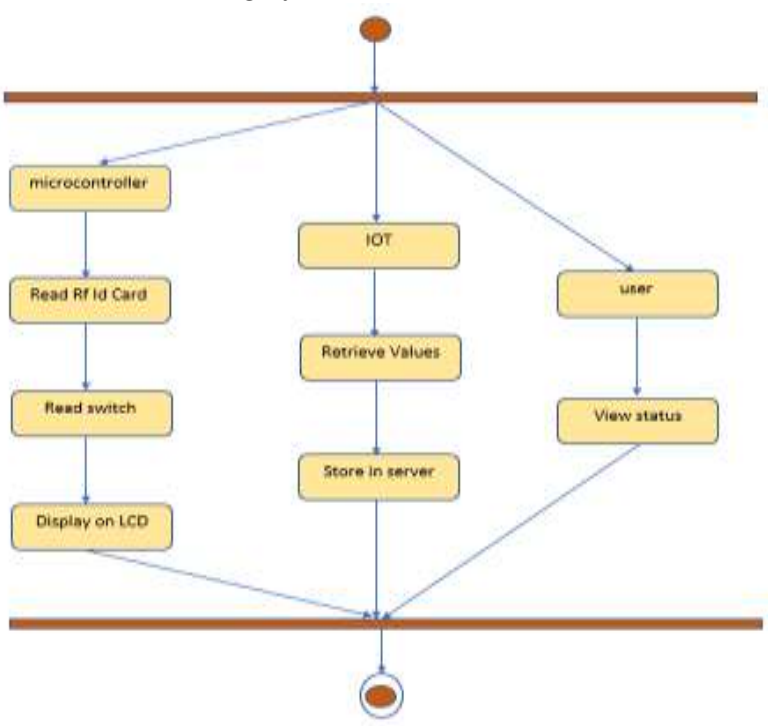

Fig 6: Activity Diagram

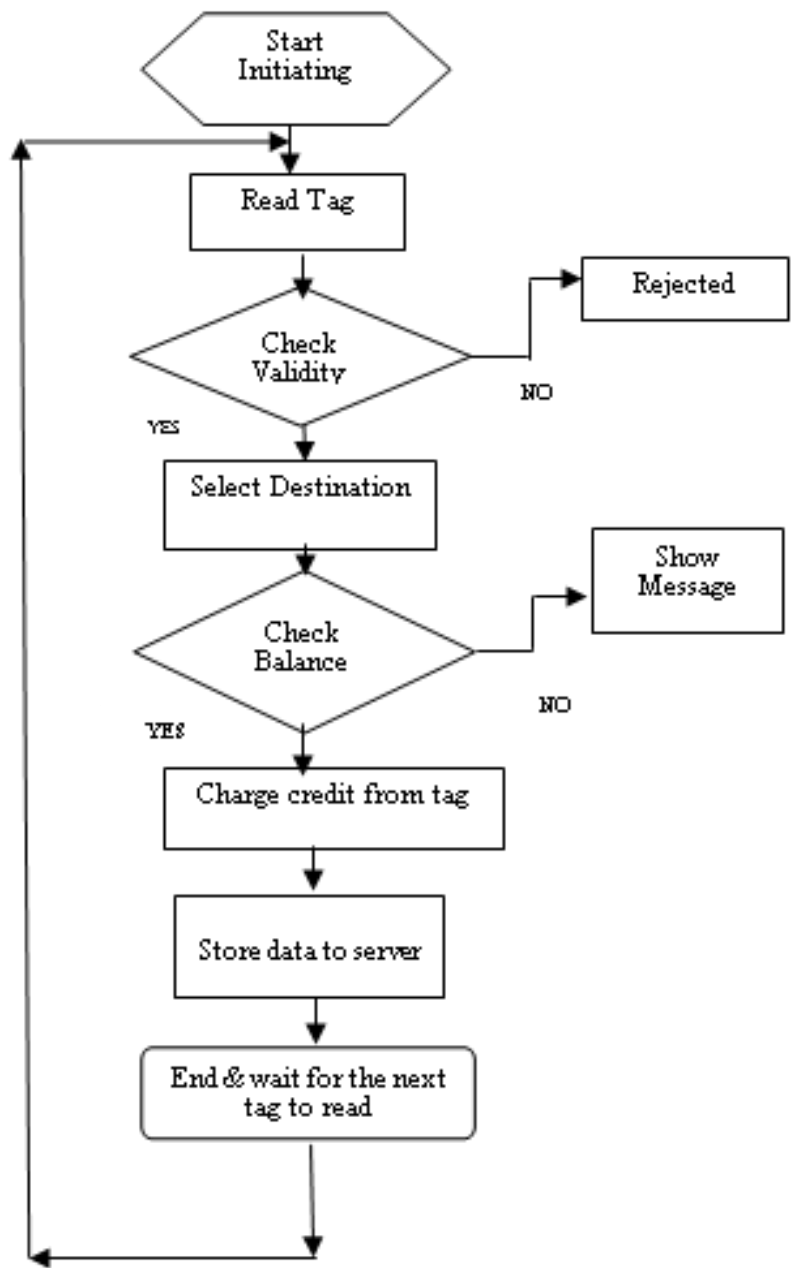

Fig 7: Flow Chart of the automatic ticketing system function

\section{EXPERIMENTAL RESULTS}

The laboratory prototype of automatic ticketing system has been tested for the functions like detecting the smart card, authentication, billing, and recharge of the smart card. The initialization of the system is shown in Fig.8. The smart card detection and authentication is carried out and shown in fig. 9 and Fig.10 respectively. The bill generation and account balance details is shown in Fig. 11.
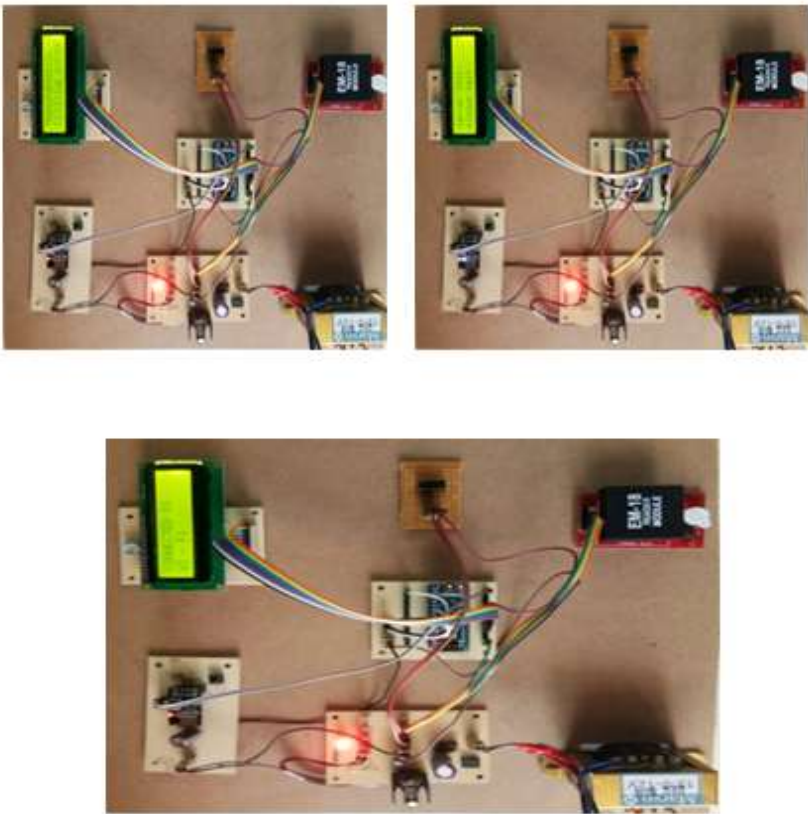

Fig.8. System initialization

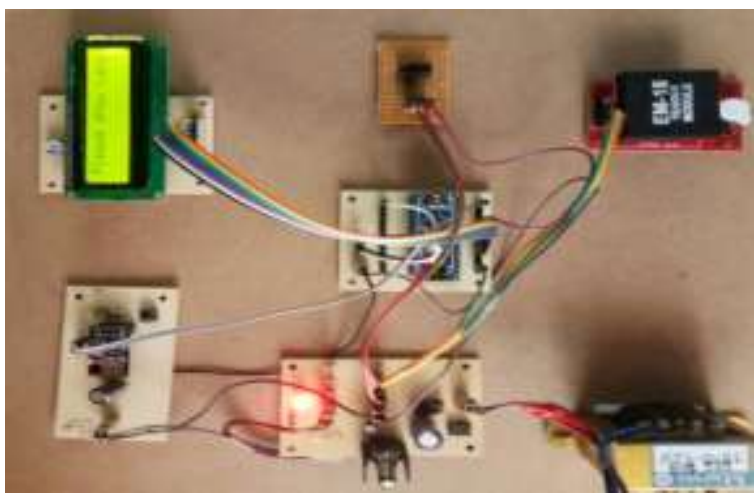

Fig.9. Detection smart card
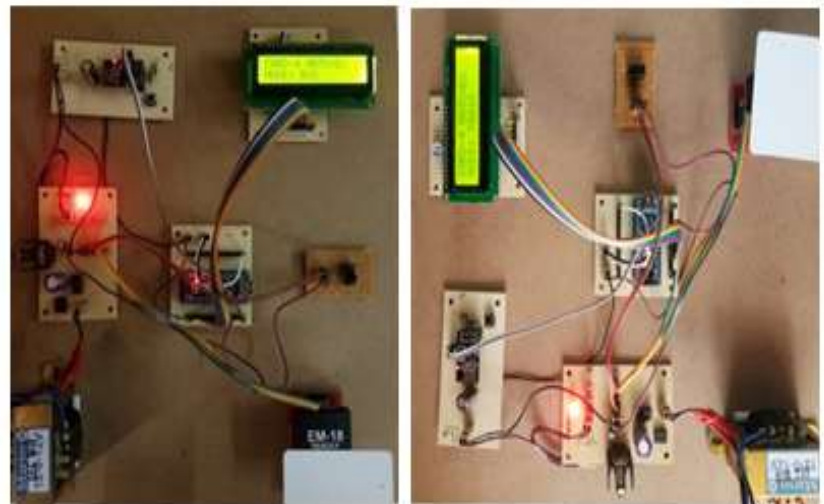

Fig.10. Authentication

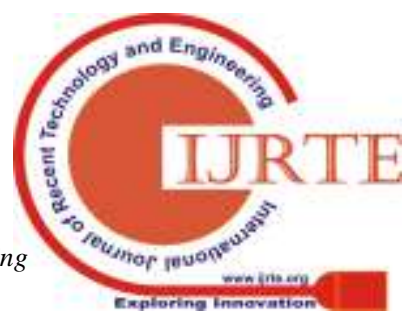



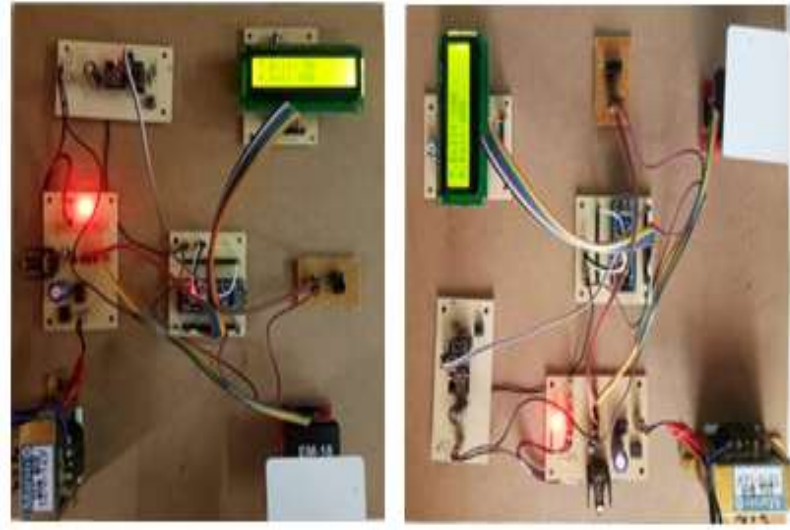

Fig.11. Bill generation

The automatic ticketing system has been verified and is functioning to meet the real time requirements.

\section{CONCLUSION}

In this paper, the development automated public transport ticketing system using micro controller, smart card and IoT is demonstrated with a laboratory prototype. This public transport ticketing system can be used to travel in any mode of transportation like Bus, Trains, Metros, and Cabs using only one smart card. The system is tested for the functions like detecting the smart card, authentication and billing. This system is completely machine-controlled and reduces the human resource. Also, it reduces the major source of financial loss due to papers. This system can be fabricated as an industry model and its application can be extended to any authenticated transport billing system.

\section{REFERENCES}

1. Anila Cyril et al, "Electronic ticket machine data analytics for public bus transport planning," in Proc. IEEEICECDS-2017,pp.3917-3923, 2017.

2. Venugopal Prasanth, Hari Prasad R., K.P. Soman, "Ticketing solutions for Indian railways using RFID technology," in Proc. IEEE-Int. Conf. on Advances in Computing, Control, and Telecommunication Technologies, pp.217-219,2009.

3. K. Finkenzeller, "RFID Handbook: Fundamentals and applications in contactless smart cards, radio frequency identification," West Sussex:ohn Wiley \& Sons, Ltd, 2010.

4. Marie-Pier Pelletier, Martin Trépanier, Catherine Morency, "Smart card data use in public transit: A literature review," ELSEVIER-Transportation Research Part C 19 (2011) 557-568,2011.

5. P. T. Blythe, "Improving public transport ticketing through smart cards," in Proc. Of the Institution of Civil Engineers -Municipal Engineer, vol. 157, no. 1, pp. 47-54, 2004.

6. Caulfield and M. O'Mahony, "Passenger requirements of a public transport ticketing system," in Proc. IEEE -Intelligent Transportation Systems, pp. 119-124 ,Sept 2005.

7. N. Mallat, M. Rossi, V. K. Tuunainen, and A. Öörni, “An empirical investigation of mobile ticketing service adoption in public transportation," Personal and Ubiquitous Computing, vol. 12, no. 1, pp. 57-65, 2008.

8. R. Widmann, S. Grünberger, B. Stadlmann, and J. Langer, "System integration of nfc ticketing into an existing public transport infrastructure," in Proc. IEEE -Near Field Communication (NFC), pp. 13-18,2012.

9. Ufuk Demir Alan, and Derya Birant, "Server-Based
Intelligent Public Transportation System with NFC," IEEE- Intelligent transportation systems magazine, pp.30-46,spring 2018.

10. Data sheet: Arduino Nano- ATmega328p.

11. User manual: Arduino IDE.

12. Data sheet: EM-18 RFID Reader.

13. Data sheet :ESP8266 Wi-Fi.

14. User manual: PHP language. 